\title{
Correction of Thickness Artifacts in EFTEM Elemental Mapping of Biological Specimens
}

\author{
M. A. Aronova, G. Zhang, and R. D. Leapman
}

Division of Bioengineering and Physical Science, ORS, National Institutes of Health, Bethesda, MD 20892

Elemental mapping in the energy filtering transmission electron microscope (EFTEM) provides important information about the distribution of bound elements in cells. For example, phosphorus is a major component of nucleic acids and mapping its distribution can reveal the arrangement of DNA in the nucleus [1]; and sulfur occurs at relatively high concentration in proteins that are rich in the amino-acids cysteine and methionine [2]. Nevertheless, the concentrations of phosphorus in sectioned cells are still typically $<1$ atomic $\%$, even in the chromatin structures of the cell nucleus, and concentrations of sulfur and other elements are lower yet. Therefore, special attention must be given to subtracting the signal from the background intensity [3]. In particular, the non-uniformity of the specimen thickness limits the reliability of the background subtraction due to changes in shape of the spectrum due to plural inelastic scattering. Previously the shape change has been taken into account using a three-window method, by fitting an inverse power law to the pre-edge background but this approach often adds an excessive amount of noise. Here, we show that it is preferable to use a two-window mapping approach and apply a precise correction for the change in shape of the background [4]. The approach is demonstrated for phosphorus mapping of a biological plastic section. Interestingly, we find that EFTEM phosphorus mapping can be performed on specimens with thicknesses much greater than had been assumed before, provided the thickness correction is applied.

To estimate the correction that must be applied to the phosphorus distribution determined by using a standard 2-window method, we model the change of shape of energy loss spectrum due to the plural inelastic scattering as a function of relative specimen thickness, $t / \lambda$, where $t$ is thickness and $\lambda$ is the inelastic mean free path. First, the single scattering intensity is determined for pure embedding medium (epon) by recording the energy-loss spectrum from a uniformly thin section and by applying the logarithmic Fourier deconvolution method. Then, plural scattering is added for different thicknesses using an inverse exponential Fourier convolution to obtain the thickness dependence of the post-edge to pre-edge intensity ratio, $k$. Having established the $t / \lambda$ dependence of the $k$ ratio, we can correct the two-window images to remove artifacts due to thickness variations. To achieve this, it is necessary to determine the $t / \lambda$ image from the logarithm of the ratio of unfiltered to zero-loss images, recorded from the same area of the specimen.

Fig. 1a shows an uncorrected phosphorus two-window map of a plastic section, in which compression during cutting produced thickness variations with $t / \lambda$ values ranging from 0.7 to 1.2 . Artifacts are evident because a single value of the $k$ ratio was used. In Fig. 1b, the thickness correction has been applied using a $t / \lambda$ dependent $k$ ratio. It is evident that the artifacts due to the thickness variation have disappeared and the image intensity reflects the phosphorus distribution in the specimen. The method can be applied to samples of thickness as great as 1.5 inelastic mean free paths, which corresponds to over $200 \mathrm{~nm}$ at a beam voltage of $300 \mathrm{kV}$. The method could be applied to mapping other elements in biological or some types of non-biological specimens. 


\section{References}

[1] D.P. Bazett-Jones et al., Science 264 (1994) 1134.

[2] R.D. Leapman et al., J. Struct. Biol. 120 (1997) 168.

[3] F. Hofer et al., Ultramicroscopy 67 (1997) 83.

[4] M.A. Aronova et al. (2006) in press.

(a) single $k$ value

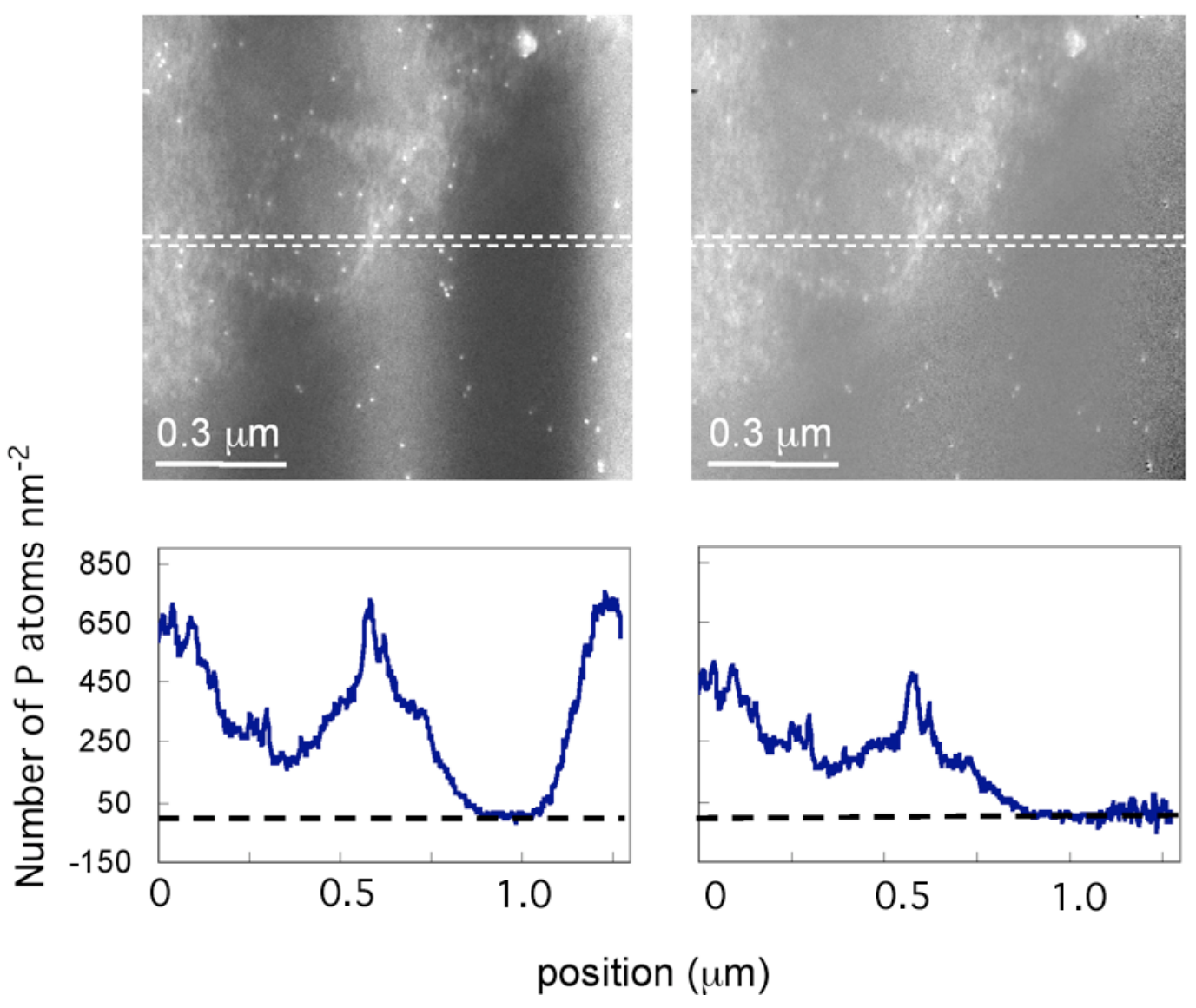

Fig. 1. Phosphorus distribution and a line profile for plastic embedded cell nucleus using (a) single $k$-value thickness correction and (b) $k$-curve correction. In (a) artifacts are due to the thickness variations. In (b) artifacts disappear and plastic embedding medium exhibits no significant phosphorus content. The EFTEM data were recorded on a $120 \mathrm{kV}$ FEI CM120 TEM equipped with a GIF100 Imaging Filter. The specimen thickness across the image area varied from a $t / \lambda$ value of 0.7 to 1.2 . 